\title{
Estudio sobre el desarrollo de protocolos familiares en Galicia
}

\author{
SUSANA BARBEITO ROIBAL, DIEGO CRESPO PEREIRA, MANUEL MARTÍNEZ CARBALLO \\ y EDUARDO GUILLÉN SOLÓRZANO \\ Departamento de Análisis Económico y Administración de Empresas \\ UNIVERSIDAD DE A CORUÑA \\ e-mail: sbar@cdf.udc.es
}

\begin{abstract}
RESUMEN
Un proyecto de investigación sobre empresa familiar gallega, financiado por la Universidade da Coruña durante los años 2004 y 2005, ha permitido analizar las respuestas, procedentes de 57 empresas familiares gallegas que facturaban en el año 2003 más de 5 millones de euros.

Uno de los aspectos recogidos en este proyecto, y que constituye el objeto de estudio de este artículo, muestra cuales son las variables que influyen en el desarrollo de protocolos familiares en las empresas familiares gallegas. La literatura sobre empresa familiar destaca su relevancia y su influencia en procesos de sucesión exitosos. Los resultados obtenidos, muestran cambios en la importancia que los empresarios familiares gallegos conceden a los protocolos, aproximándose cada vez más al nivel de relevancia que la literatura ha venido concediendo a los protocolos familiares en la última década.
\end{abstract}

Palabras clave: Protocolo familiar; empresa familiar; relaciones familia-empresa.

\section{A Study of Galician Family Protocols}

\begin{abstract}
A research project on Galician family business, financed by the Coruña University during years 2004 and 2005 , has allowed to analyze the answers, coming from 57 Galician familiar companies that invoiced in year 2003 more than 5 million euro. One of the aspects gathered in this project, and that constitutes the object of study of this article, shows as it is the importance that the Galician family owners grant to the planning for the succession process. Literature on familiar business emphasizes its relevance and its influence in exits successful processes. The obtained results, more and more show changes in the importance that the Galician family owners grant to our aim of study, coming near at the relevance level that Literature has come granting to the succession process in the last decade.
\end{abstract}

Keywords: Family Business; Family and Business Relationships; Family Protocol.

Clasificación JEL: L20.

Artículo recibido en junio de 2006 y aceptado para su publicación en febrero de 2008.

Artículo disponible en versión electrónica en la página www.revista-eea.net, ref. อ-26216. 


\section{INTRODUCCIÓN}

El tópico de la sucesión ha dominado los estudios científicos en el campo de la empresa familiar en los últimos años. Sorprendentemente, el análisis sobre cuáles son las variables que influyen en el desarrollo de protocolos familiares ocupa un lugar secundario, limitándose la mayoría de la bibliografía más reciente a análisis jurídicos, Sancho (2005), González de Zulueta y Paisant (2007); y artículos de divulgación. Y todo esto ocurre mientras la realidad muestra que, a pesar de los cambios en la legislación de los distintos países, de las ayudas que financian parte del coste de estos protocolos en distintas comunidades autónomas españolas,... los empresarios familiares siguen siendo reacios a su desarrollo.

La relevancia y actualidad del objeto de estudio de este artículo en nuestro país, es destacada por Gortázar (2004) al afirmar que: en 2001 y a instancia de una moción del Senado, se constituyó un artículo en el seno de la Comisión de Hacienda del Senado para que emitiera un informe. Dicho informe propuso: 1. Recomendar que por parte de las Administraciones Públicas se adopten políticas de información y comunicación para el desarrollo y mejora de la gestión en las empresas familia$\operatorname{res}^{1}, 2$. Recomendar a las empresas familiares la formalización de un protocolo familiar, al considerarlo el instrumento más adecuado para regular la problemática de las empresas familiares.

Es también destacable la disposición final segunda, apartado 3 de la Ley 7/2003, de 1 de Abril, de la sociedad limitada nueva empresa que dispone que reglamentariamente se establecerán las condiciones, forma y requisitos para la publicidad de los protocolos familiares, así como, en su caso, el acceso al registro mercantil de las escrituras públicas que contengan cláusulas susceptibles de inscripción.

Posteriormente el Real Decreto 171/2007, de 9 de Febrero, regulará la publicidad de los protocolos familiares.

Gortázar (2004) nos recuerda que no sólo en España están teniendo lugar importantes cambios en la legislación aplicable a las empresas familiares, sino que también las instituciones europeas se han preocupado desde hace algunos años por la continuidad de las pequeñas y medianas empresas, muchas de ellas de carácter familiar como motor de la economía europea. Así cabe mencionar la Recomendación de la Comisión Europea, 94/1069/CE, de 7 de Diciembre de 1994, que reconocía las causas de derecho sustantivo y fiscal que dificultaban la transmisión de la empresa, sobre todo mortis causa, y aconsejaban reformas dirigidas a simplificar el Derecho para facilitar la continuidad y supervivencia de las pymes; y las Recomendaciones de Forum de Lille (celebrado el 3 y 4 de febrero de 1997) que se refieren a medidas legislativas que deberían adoptarse, tales como la supresión de la prohibición de los pactos sucesorios y la suscripción del protocolo familiar.

\footnotetext{
${ }^{1}$ Así en Cataluña, el Departament d'Indústria, Comerç i Turisme, mediante Resolució ICT/3085/2002, de 16 de Octubre, adoptó una política de ayudas a las empresas familiares para la realización de planes de sucesión (codi 121). DOGC núm. 3751, de 30 de Octubre de 2002.
} 
A pesar de todos estos cambios en materia legislativa, existen pocos estudios sobre el número de protocolos familiares que se han redactado en España. Uno de estos estudios, llevado a cabo por Amat en 1998, analizó 100 empresas familiares españolas, llegando a las siguientes conclusiones: el 10\% de las empresas analizadas disponían de un protocolo familiar y un $20 \%$ tenían la intención de completarlo antes del 2000. Estos datos muestran la escasa incidencia de los protocolos familiares en las empresas familiares españolas.

A continuación se llevará a cabo una breve revisión bibliográfica, de la literatura consultada, sobre los protocolos familiares y también se hará mención a la metodología utilizada en este trabajo. Posteriormente se expondrán los resultados obtenidos en este estudio sobre las variables que influyen en la redacción de protocolos familiares en las empresas familiares gallegas.

\section{REVISIÓN BIBLIOGRÁFICA}

En las últimas décadas se han diagnosticado muchos problemas que afectan a la empresa familiar pero, sin duda el mayor reto es lograr su supervivencia garantizar su continuidad y sucesión, debe ser el objetivo de todos los que nos dedicamos, desde distintos ámbitos al estudio o asesoramiento de este tipo de organizaciones. Uno de los grandes instrumentos que puede ayudar a alcanzar este objetivo es la redacción de protocolos familiares.

Nos gustaría destacar la inexistencia de estadísticas nacionales sobre empresa familiar quizás debido como sugieren Chrisman et al. (1998) a la no existencia de una definición sobre empresa familiar aceptada mayoritariamente. No sólo no existe una definición que nos satisfaga a los investigadores, sino que ni siquiera existe en nuestro ordenamiento jurídico. A pesar de ello, como destacan González de Zulueta y Paisánt (2007), la doctrina sí se ha ocupado de este tipo de empresas desde el punto de vista conceptual y organizativo, siendo quizá la caracterización recogida por el Real Decreto 171/2007 una de las más recientes. En el mismo se define a las empresas familiares como "aquéllas en las que la propiedad o el poder de decisión pertenecen, total o parcialmente, a un grupo de personas que son parientes consanguíneos o afines entre sí".

Es también destacable que países de nuestro entorno sí tienen un concepto legal de empresa familiar, por ejemplo en el ordenamiento italiano, el artículo 230 bis de la Ley de Reforma del Derecho de Familia, de fecha 19 de Mayo de 1975, define la empresa familiar como "aquélla donde la familia presta de manera continuada su trabajo y colaboran los familiares, entendiéndose por familiares al cónyuge, los parientes dentro del tercer grado y los afines dentro del segundo grado".

El Real Decreto 171/2007, entiende que un protocolo familiar es "aquel conjunto de pactos suscritos por los socios entre sí o con terceros con los que guardan vínculos familiares respecto de una sociedad no cotizada en la que tengan un interés común en orden a lograr un modelo de comunicación y consenso en la toma de decisiones para regular las relaciones entre familia, propiedad y empresa que afec- 
tan a la entidad". Siendo ésta una definición bastante próxima a las que mostraremos en este artículo y que han sido obtenidas de la bibliografía cónsultada, Ward, (1993) y Fernández-Tresguerres (2002).

A lo largo de este artículo se tratarán distintos aspectos referentes a las variables que influyen en la redacción de protocolos familiares en las empresas familiares gallegas, así estos aspectos se recogen en tres grandes núcleos temáticos: i) influencia de características generales del máximo responsable en el desarrollo de protocolos familiares, ii) normas que regulan las relaciones familia empresa, y, iii) desarrollo de protocolos familiares. Un breve repaso a la literatura sobre empresa familiar muestra la importancia de los mismos.

i) Influencia de características generales del máximo responsable en el desarrollo de protocolos familiares. A lo largo de este punto se tratarán distintos aspectos referentes a la relación existente entre las características de los sucesores en las empresas familiares gallegas recogidas en tres grandes bloques: (i) La competencia; (ii) Las relaciones familiares y; (iii) La posición en la familia y el desarrollo de protocolos familiares en las mismas. Un breve repaso a la literatura sobre empresa familiar, muestra la importancia de algunas de las características enumeradas con anterioridad. La literatura hace referencia a aspectos como: el nivel de formación o la experiencia profesional, tanto en el propio negocio familiar como la experiencia externa, Schiff Estess (2001). Parece evidente que el nivel de formación del sucesor o su experiencia laboral, serán indicativos a priori, de su nivel de conocimientos, de su capacidad para enfrentarse a los problemas y de resolverlos con acierto, o incluso de su receptividad al I+D+i. Muchos autores han hecho referencia a estos aspectos, Lansberg \& Astrachan (1994); Barnes (1998) o Cabrera (1989). Otro aspecto relevante sería el criterio utilizado para elegir sucesor, así el criterio de primogenitura, es el que históricamente ha tenido una mayor aceptación a la hora de transferir el poder en las empresas familiares, en él prima claramente el componente emocional sobre el componente racional, puesto que basa la sucesión en la empresa familiar, en el acceso al puesto de máximo responsable del primer hijo varón, Sharma et al. (1997). Es fácilmente constatable que fuertes tradiciones han apoyado la primogenitura en las empresas de propiedad familiar, es decir, los varones primogénitos tienen preferencia en materia de herencia, Olson (2001); Rosenblatt et al. (1985); Stavrou (1999). Cabrera (1998), sugiere que una de las razones por las cuales existe esta reticencia al cambio de criterio es debida a que se trata, muchas veces, de una asunción automática que evita a los padres la desagradable situación de comparar y elegir entre los hijos.

ii) Normas que regulan las relaciones familia empresa. Con respecto a las relaciones familiares, la literatura destaca la importancia de las buenas relaciones del sucesor tanto con el actual máximo responsable, como con el resto de miembros de la familia. Parece evidente que la buena relación sucesor-actual máximo responsable puede ser la llave de un proceso sucesorio exitoso, Barnes y Hershon (1976), Handler, (1992). En base a ello cabría preguntarse si existen 
normas que regulan aspectos como: la política de distribución de dividendos, el mantenimiento del buen clima familiar, la existencia de un consejo de familia... Y si los empresarios familiares gallegos han recogido estas normas en un documento aceptado por el conjunto familiar.

iii) Desarrollo de protocolos familiares. Tradicionalmente el contenido del protocolo familiar se ha venido considerando como un conjunto de metas para la familia y la empresa, y en las reglas de actuación a seguir en las relaciones entre las personas de los citados sistemas. La esencia del protocolo familiar es un acuerdo veraz sobre dichas metas y reglas unido al compromiso de empeñarse en su cumplimiento. Es una declaración de intenciones consensuada por todos los miembros de las generaciones de la familia. Para Ward (1993) un protocolo familiar, desde el punto de vista de la empresa, debería ayudar a profesionalizar los procesos de dirección estratégica, y a institucionalizar en la empresa los valores básicos de la familia. Desde el punto de vista de la familia, debería ayudar a promover la unidad y armonía de sus miembros, a profesionalizarles como propietarios de una empresa, y a conservar en la familia los valores que hacen fuerte a la empresa familiar. Es, por tanto, una herramienta dinámica (como recoge nuestro ordenamiento jurídico RD 171/2007) que se revisa periódicamente y que consta de dos partes: los principios o reglas del juego y las decisiones operativas. El objetivo básico del protocolo familiar es el mantenimiento de la empresa familiar; pretende por tanto: 1. Defender e incrementar el patrimonio de la familia, evitando la dispersión del capital, 2. Proteger la estrategia de las empresas del grupo familiar de la intromisión abusiva de la familia en la gestión, ello implica limitar el acceso a la empresa de los miembros de la familia y su número dentro del consejo de administración y, 3. Evitar o ayudar a superar las crisis estructurales típicas de las empresas familiares.

Ward (1993) incide en la importancia del protocolo familiar como medio que favorece la profesionalización del proceso de dirección estratégica de la empresa familiar. Coincidiendo con numerosos autores, Sharma et al. (1997); Drozdow and Carrol (1997); Fernández (1993); Vilanova et al. (1999); Cabrera (1998); en la importancia de la aplicación de los principios de dirección estratégica a distintos ámbitos de la empresa familiar.

Más recientemente, el Real Decreto 171/2007, de 9 de Febrero, regula la publicidad de los protocolos familiares, en España, reconociendo que "la cultura del protocolo familiar, shareholders agreement, es considerada una garantía adicional para terceros, inversores y acreedores, además de para los propios socios, al dotar de previsibilidad el relevo generacional en la sociedad."

A pesar de todo lo dicho anteriormente, existen también autores como Pont Clemente (1998) que son escépticos sobre las bondades del protocolo familiar, ya que consideran que la mayor parte de las teorías sobre el mismo han nacido para resolver el problema de la falta de identidad de la empresa familiar con propiedad dividida, asumiendo como dogma la necesidad de mantenimiento de la unidad y 
descartando cualquier análisis crítico sobre el reparto igualitario de las alícuotas de propiedad. Es decir, el protocolo pretende solucionar un problema enormemente complejo, afrontando sus consecuencias, pero evitando, escrupulosamente, atacar sus raíces. Nueno (1999), en una línea similar a la de Pont Clemente, considera que un protocolo familiar no se trata más que de un "papel" sin eficacia legal, redactado al gusto de quien lo encargó.

Desde este punto de vista se podría preguntar a los empresarios familiares gallegos, si se han planteado alguna vez la necesidad de desarrollar un protocolo familiar y, en caso afirmativo, si tienen previsto desarrollarlo en breve, si han necesitado ayuda para llevar a cabo este proceso y, si no lo han desarrollado cuál ha sido la causa.

\section{METODOLOGÍA}

El método dominante en la investigación sobre empresa familiar consiste en utilizar encuestas a gran escala entre una muestra representativa, Bird, et al. (2002); Davidsson et al. (2001); Dyer\&Sánchez (1998); Fillis (2001); Grant \& Perren (2002).

Metodológicamente, el punto de partida de este trabajo son las empresas que forman parte de la base de datos ARDÁN a la cual se le han aplicado unas restricciones iniciales: 1. Restricción de carácter geográfico, donde su ámbito se redujo a Galicia y, 2. Restricción por volumen de facturación, ciñéndonos a aquellas empresas que facturaron en el año 2003 más de 5 millones de euros. Con estas restricciones ARDÁN contenía 1243 empresas en su base de datos. Posteriormente, esta base de datos se somete a un proceso de clasificación separando los distintos grupos empresariales gallegos en familiares y no familiares. Para ello, se utilizan distintos indicadores de su carácter de empresa familiar como: el año de fundación de la empresa, apellidos concordantes en sus consejos de administración o el conocimiento personal de su condición de empresa familiar. Esta clasificación se completa posteriormente con una pregunta en la encuesta, que requiere de los propios empresarios una confirmación del carácter familiar de su empresa.

Estimamos, a partir del proceso anterior, que la base de datos ARDAN con las restricciones expuestas recoge a 467 empresas familiares; 687 empresas no familiares y 89 que no se han logrado clasificar. A su vez se procede a depurar la base de datos quedando reducida a 1065 empresas, de las cuales 460 son empresas familiares y 605 son empresas no familiares.

A estas 1065 empresas se les envía, en Marzo de 2005, una encuesta abarcando distintos aspectos que en lo que a empresas familiares se refiere hacen referencia a la caracterización del sucesor y otros aspectos relacionados con la situación de las mismas (proceso de sucesión, protocolos familiares, implantación de sistemas de gestión de la calidad...). Con el Software SPSS 14.0 se procede al tratamiento estadístico de los datos obtenidos. El resumen de las respuestas recibidas recoge 57 empresas familiares gallegas, lo cual supone aproximadamente una tasa de respuesta del $12 \%$ que la hace significativa 
A partir de los datos recogidos en la tabla 1 se podría caracterizar a las empresas familiares gallegas, que han respondido a la encuesta: son empresas que se fundan como media en 1974, con una facturación entre 10 y 50 millones de euros, con una platilla entre 50 y 249 empleados, localizadas en la costa atlántica (A Coruña y Pontevedra) en el $94 \%$ de los casos y están mayoritariamente $(45,83 \%)$ en primera generación.

TABLA 1

Caracterización de las empresas familiares que respondieron a la encuesta.

\begin{tabular}{|c|c|c|c|}
\hline \multicolumn{4}{|c|}{ Empresas Familiares Gallegas } \\
\hline $\begin{array}{l}\text { Año de } \\
\text { fundación }\end{array}$ & $\begin{array}{l}\text { Máximo: } 1997 \\
\text { Mínimo: } 1930 \\
\text { Media: } 1974,32 \\
\text { Desv. Típica: } \\
\text { 14,632 }\end{array}$ & $\begin{array}{l}\text { Facturación (2003): } \\
\text { (millones de } € \text { ) }\end{array}$ & $\begin{array}{l}5-10: 27,1 \% \\
10-50: 33,6 \% \\
+50: 39,6 \%\end{array}$ \\
\hline $\mathrm{N}^{\circ}$ empleados & $\begin{array}{l}0-9: 2,1 \% \\
\text { 10-49: } 22,9 \% \\
50-249: 50,0 \% \\
+249: 18,8 \%\end{array}$ & $\begin{array}{l}\text { Generación al frente de } \\
\text { la empresa }\end{array}$ & $\begin{array}{l}\text { Primera: } 45,83 \% \\
\text { Segunda: } 39,58 \% \\
\text { Tercera: } 12,5 \% \\
\text { Otras: } 2,08 \%\end{array}$ \\
\hline $\begin{array}{l}\text { Ámbito } \\
\text { geográfico }\end{array}$ & $\begin{array}{l}\text { Pontevedra: } 45,8 \% \\
\text { A Coruña: } 47,9 \% \\
\text { Orense: } 4,2 \% \\
\text { Lugo: } 2,1 \%\end{array}$ & Son grupos exportadores & $\begin{array}{l}\text { Sí: } 30,8 \% \\
\text { No: } 69,2 \%\end{array}$ \\
\hline \multicolumn{3}{|c|}{$\%$ ventas que se destina a la exportación } & $\begin{array}{l}<25 \%: 55,9 \% \\
25-50 \%: 11,8 \% \\
50-75 \%: 14,7 \% \\
>75 \%: 17,6 \%\end{array}$ \\
\hline
\end{tabular}

\section{RESULTADOS OBTENIDOS}

El estudio sobre las variables que inciden en el desarrollo de protocolos familiares en las empresas familiares gallegas se ha centrado, en este artículo, en torno a cuatro núcleos temáticos: i) grado de desarrollo de los protocolos familiares en Galicia ii) relación existente con las características de la empresa iii) influencia de características del máximo responsable en el desarrollo de protocolos familiares, iv) asociación con el ámbito de aplicación de las normas que regulan las relaciones familia empresa. En cuanto al primer apartado, se exponen los porcentajes de respuestas a las diversas preguntas planteadas para caracterizar el grado de implantación de los protocolos familiares. Para el estudio de las otras carácterísticas de las empresas consideradas se ha realizado un análisis de asociación de las respuestas dadas con respecto a la existencia de un protocolo familiar. Según el aspecto recogido en la pregunta la respuesta puede estar codificada como una variable categórica u ordinal. El grado de asociación se calculará en cada caso según el coeficiente correspondiente. 
El caso de una variable dicotómica frente a otra variable dicotómica, por ejemplo las respuestas tipo sí/no, se ha estudiado mediante el coeficiente de correlación phi. La $V$ de Crámer se ha empleado cuando, siendo ambas variables nominales, alguna de ellas es politómica. Para la pregunta sobre el porcentaje de participación del grupo familiar (codificada en tres posibles respuestas: menor al 50\%, superior al 50\% y $100 \%$ ), como éste aspecto está tratado como una variable ordinal, se ha empleado el coeficiente de correlación por rangos de Spearman para determinar la asociación con respecto a la existencia o no de protocolo. Para aplicar dicho Coeficiente, se ha asignado un orden a las respuestas sobre la existencia de protocolo tal que se considera 0 la no existencia y 1 la existencia. Por último, para los dos casos de comparación de la existencia de protocolos familiares con respecto a variables cuantitativas (la edad de la empresa y la facturación), se ha empleado la medida Eta.

Los contrastes que llevados a cabo para la verificación de si el coeficiente de asociación estimado es significativo, se han realizado con una confianza del $95 \%$, lo que se corresponde con un p-valor de 0,05 .

i) Grado de desarrollo de los protocolos familiares en Galicia. En relación con el desarrollo de protocolos, el estudio revela que en Galicia sólo un 56\% se plantea su necesidad y solo un $30 \%$ de los empresarios familiares gallegos han desarrollado protocolos familiares y éstos mayoritariamente han necesitado ayuda externa para poder desarrollarlo.

Quizá a modo de resumen, destacar que todavía queda mucho por hacer y, a tenor de las respuestas ofrecidas por los responsables de las empresas familiares gallegas, una de las líneas de actuación sería claramente formativa, puesto que todavía un $34,5 \%$ de los empresarios marcan como razón por la cual no han desarrollado un protocolo familiar, la falta de conocimiento. 
TABLA 2

Desarrollo de los protocolos familiares en la empresa familiar gallega.
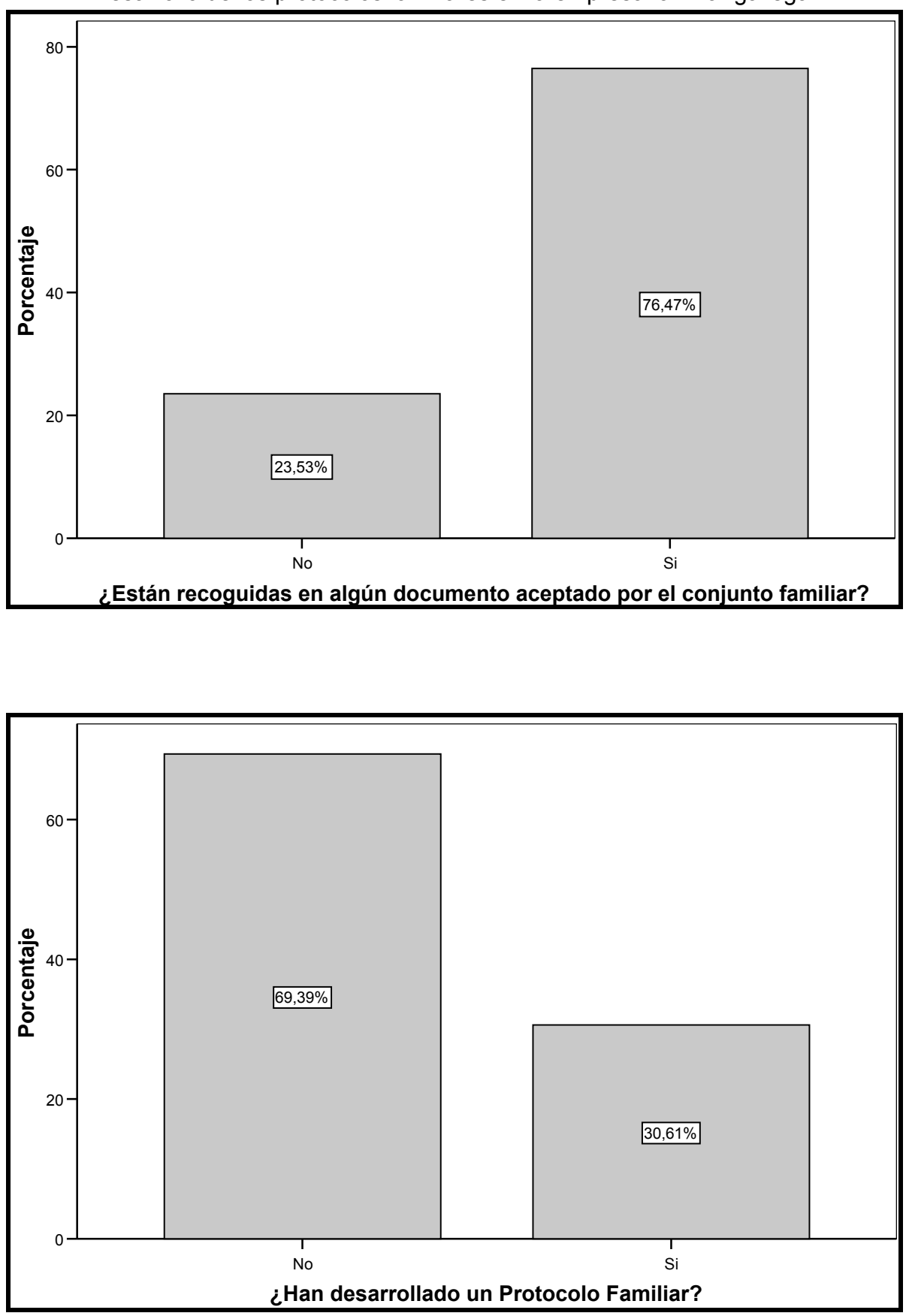
TABLA 2 (continuación)

Desarrollo de los protocolos familiares en la empresa familiar gallega.
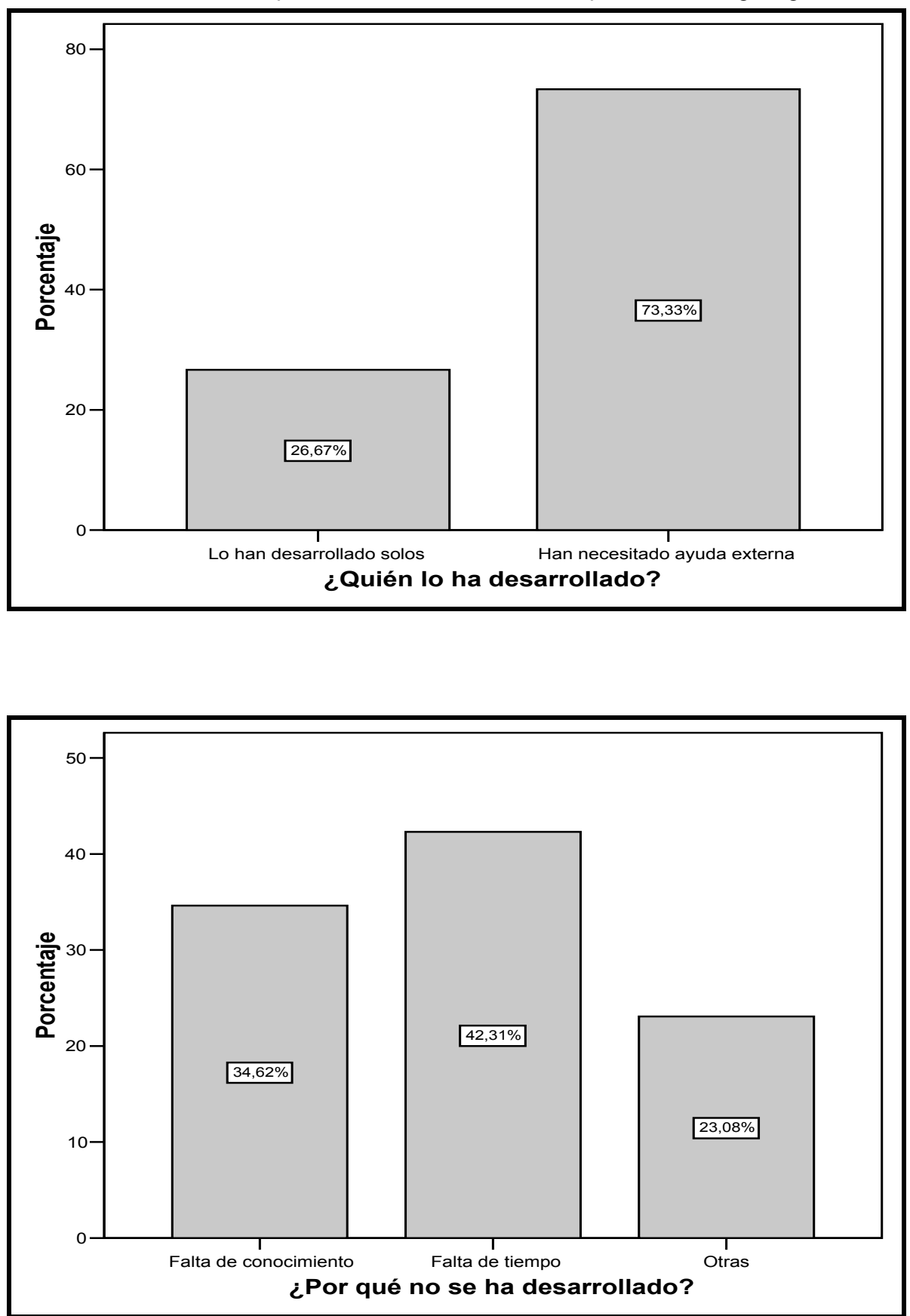
TABLA 2 (continuación)

Desarrollo de los protocolos familiares en la empresa familiar gallega.
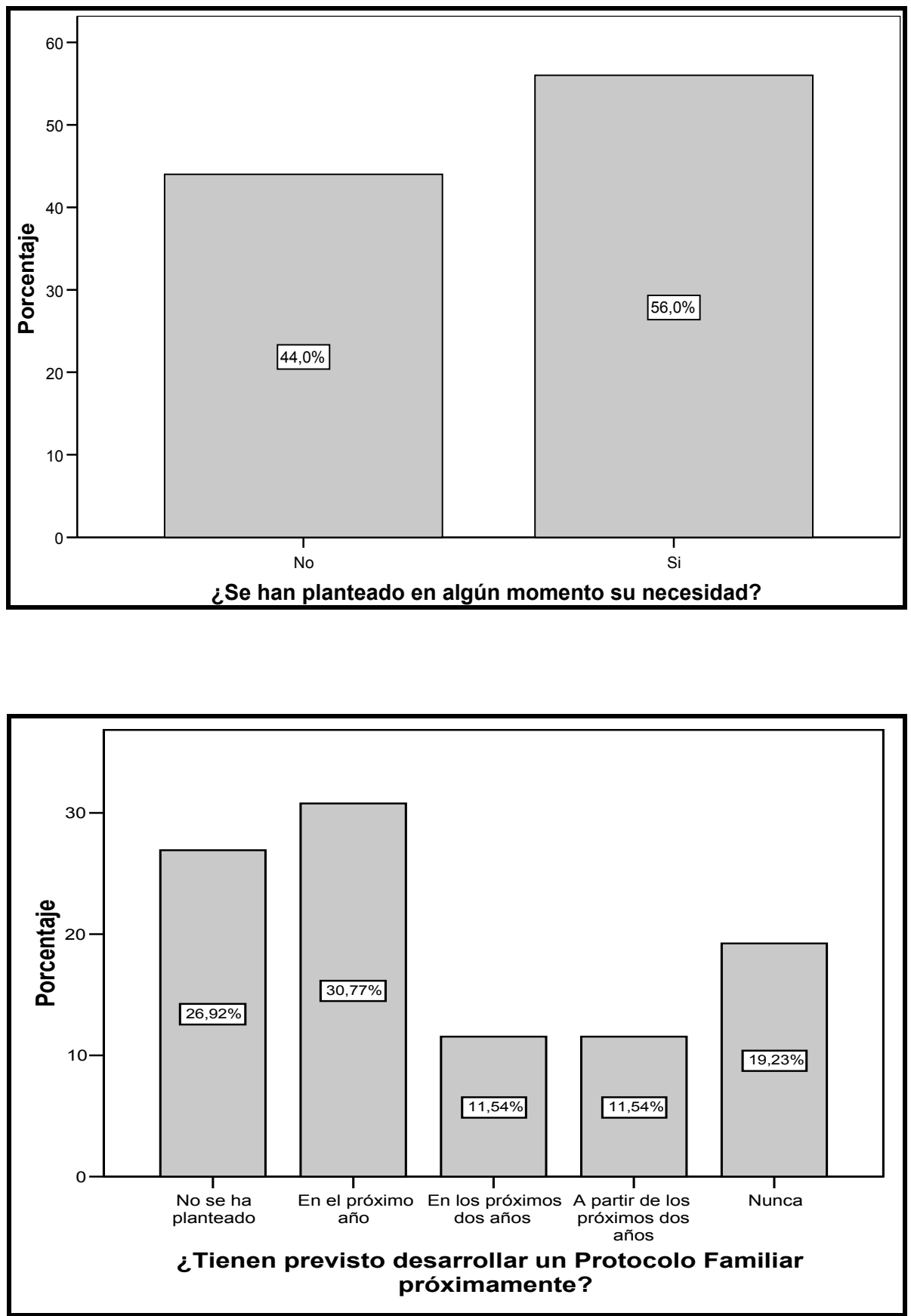
ii) Relación existente con las características de la empresa.

TABLA 3

Desarrollo de protocolos familiares frente a las características de la empresa.
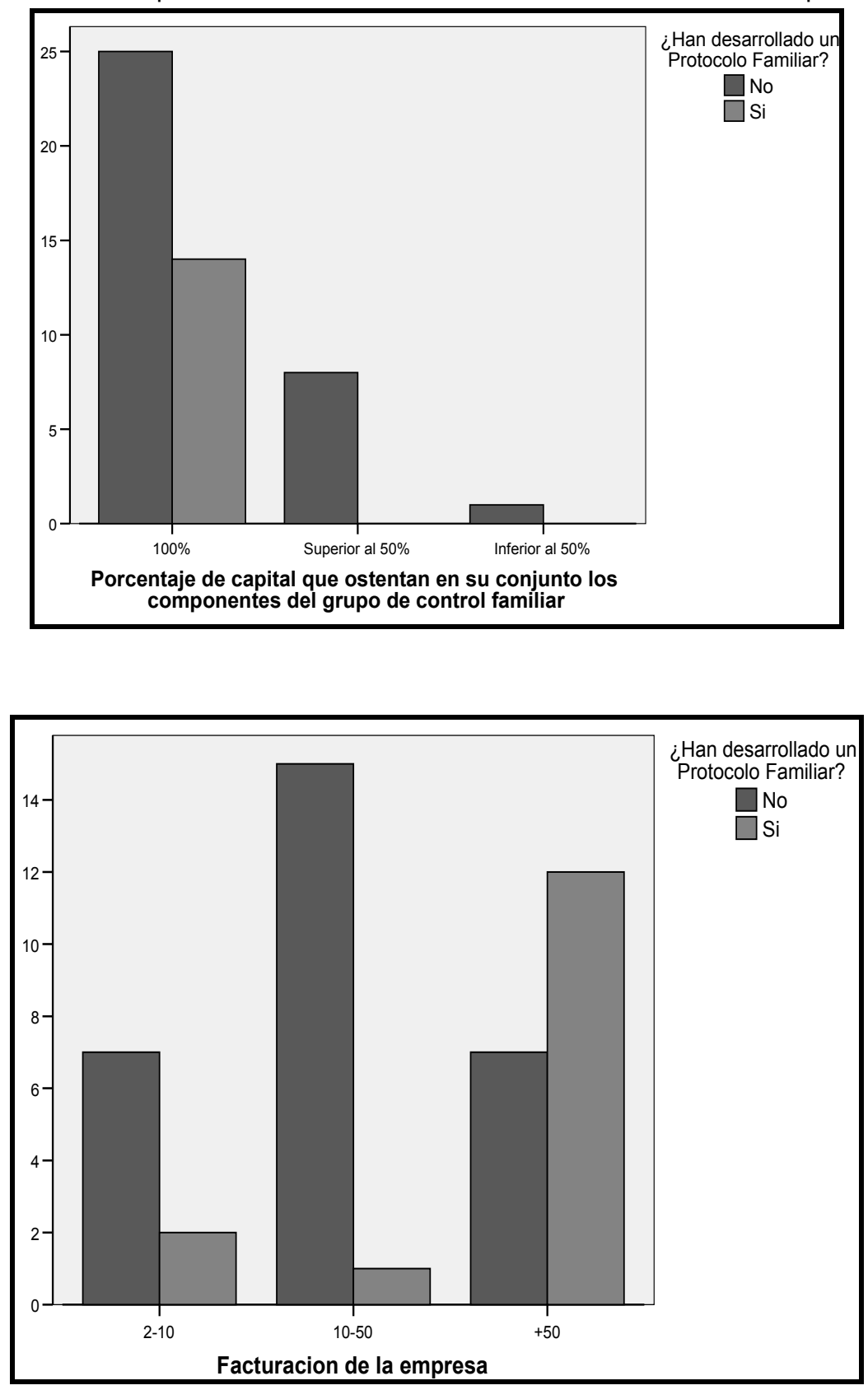
TABLA 3 (continuación)

Desarrollo de protocolos familiares frente a las características de la empresa.

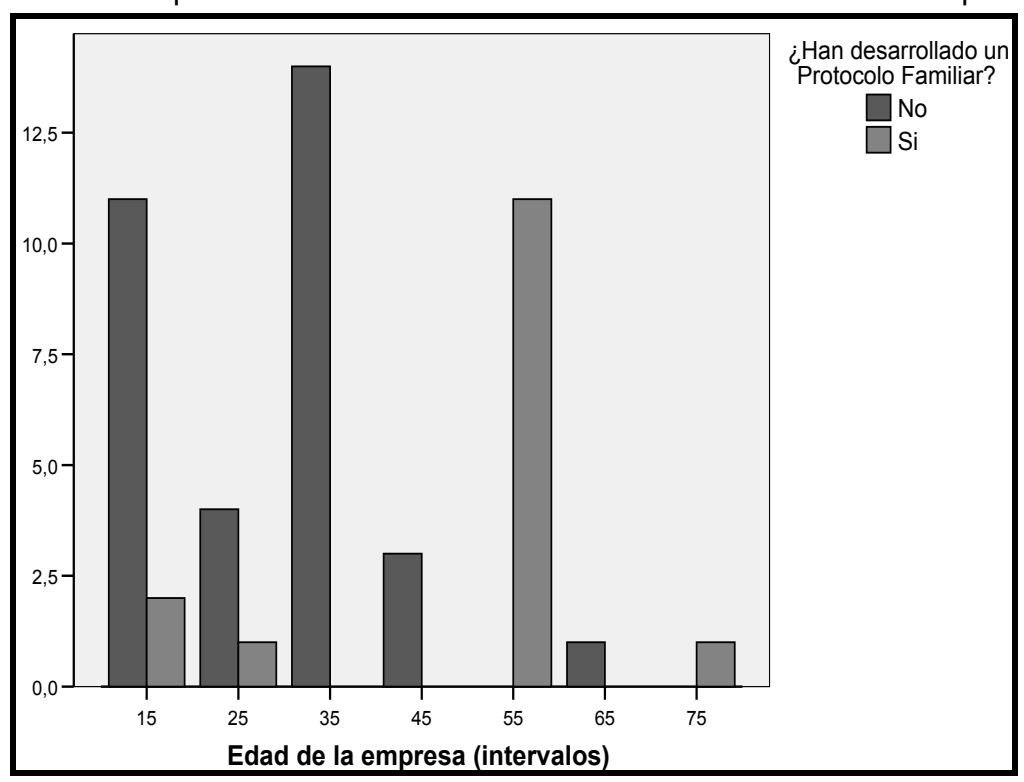

Se ha encontrado asociación entre distintas características de la empresa y la existencia de protocolos familiares. Así ocurre que a mayor grado de participación del grupo familiar en el capital de la empresa, mayor es el desarrollo de protocolos familiares. De hecho en todas las empresas en las que se ha desarrollado un protocolo familiar, el grupo familiar ostentaba el 100\% del capital. El coeficiente de correlación por rangos de Spearman es de 0,308, lo que indica que, efectivamente, hay un cierto grado de asociación en el sentido citado.

Por otro lado, se detectó una asociación entre la mayor edad de la empresa y la existencia del protocolo, ya que la medida eta para la variable "existencia de protocolo" dependiente de la edad de la empresa es de 0,871. Lo mismo se puede decir, en menor grado, para la facturación de la empresa. La medida eta para "existencia de protocolo" dependiente de la facturación fue de 0,548 , en el sentido de que a mayor facturación se da una mayor existencia de protocolos. 
TABLA 4

Asociación entre las características de la empresa y el desarrollo de protocolos familiares.

\begin{tabular}{|l|c|}
\hline \multicolumn{1}{|c|}{ Característica } & Coeficiente eta \\
\hline $\begin{array}{l}\text { Edad de la empresa dependiente, existencia de protocolo } \\
\text { familiar independiente. }\end{array}$ & 0,567 \\
\hline $\begin{array}{l}\text { Existencia de protocolo familiar dependiente, edad de la em- } \\
\text { presa independiente }\end{array}$ & 0,871 \\
\hline $\begin{array}{l}\text { Intervalo de facturación de la empresa dependiente, existen- } \\
\text { cia de protocolo familiar independiente. }\end{array}$ & 0,413 \\
\hline $\begin{array}{l}\text { Existencia de protocolo familiar dependiente, intervalo de } \\
\text { facturación de la empresa independiente. }\end{array}$ & 0,548 \\
\hline
\end{tabular}

TABLA 5

Asociación entre el porcentaje de capital que ostentan los miembros del grupo familiar y la existencia de protocolo.

\begin{tabular}{|l|c|c|c|}
\hline Característica & $\begin{array}{c}\text { Correlación } \\
\text { Spearman }\end{array}$ & Significación & $\begin{array}{c}\text { Asociación con la } \\
\text { existencia de un protocolo } \\
\text { familiar }\end{array}$ \\
\hline $\begin{array}{l}\text { Porcentaje del capital } \\
\text { que ostentan los } \\
\text { miembros del grupo } \\
\text { familiar }\end{array}$ & 0,308 & 0,033 & $\begin{array}{l}\text { Mayor \% capital - Existencia } \\
\text { de protocolo familiar }\end{array}$ \\
\hline
\end{tabular}

iii) Influencia de características generales del máximo responsable en el desarrollo de protocolos familiares. Se llevó a cabo un análisis de asociación con la variable: ¿han desarrollado un protocolo familiar?, con otras variables relacionadas con este núcleo temático. La tabla 6 , muestra la importancia de los aspectos considerados, ordenados según el grado de asociación obtenido por la $V$ de Crámer, ya que se trata de preguntas cuya respuesta está codificada como una variable politómica, como puede ser el nivel de formación, la experiencia profesional del máximo responsable o, incluso, algunas características que rodean la elección del sucesor a la hora de que el máximo responsable desarrolle un protocolo familiar en la empresa. Así los análisis efectuados muestran que variables como el nivel de formación o estudios del máximo responsable, la experiencia laboral, el tipo de supervisión que se ha seguido en el proceso de aprendizaje, la existencia de presiones para su incorporación a la empresa familiar o, la existencia de normas que regulen las relaciones familia empresa, guardan relación con el desarrollo de protocolos familiares en las empresas gallegas. 
TABLA 6

Desarrollo del protocolo familiar frente a las características del máximo responsable.
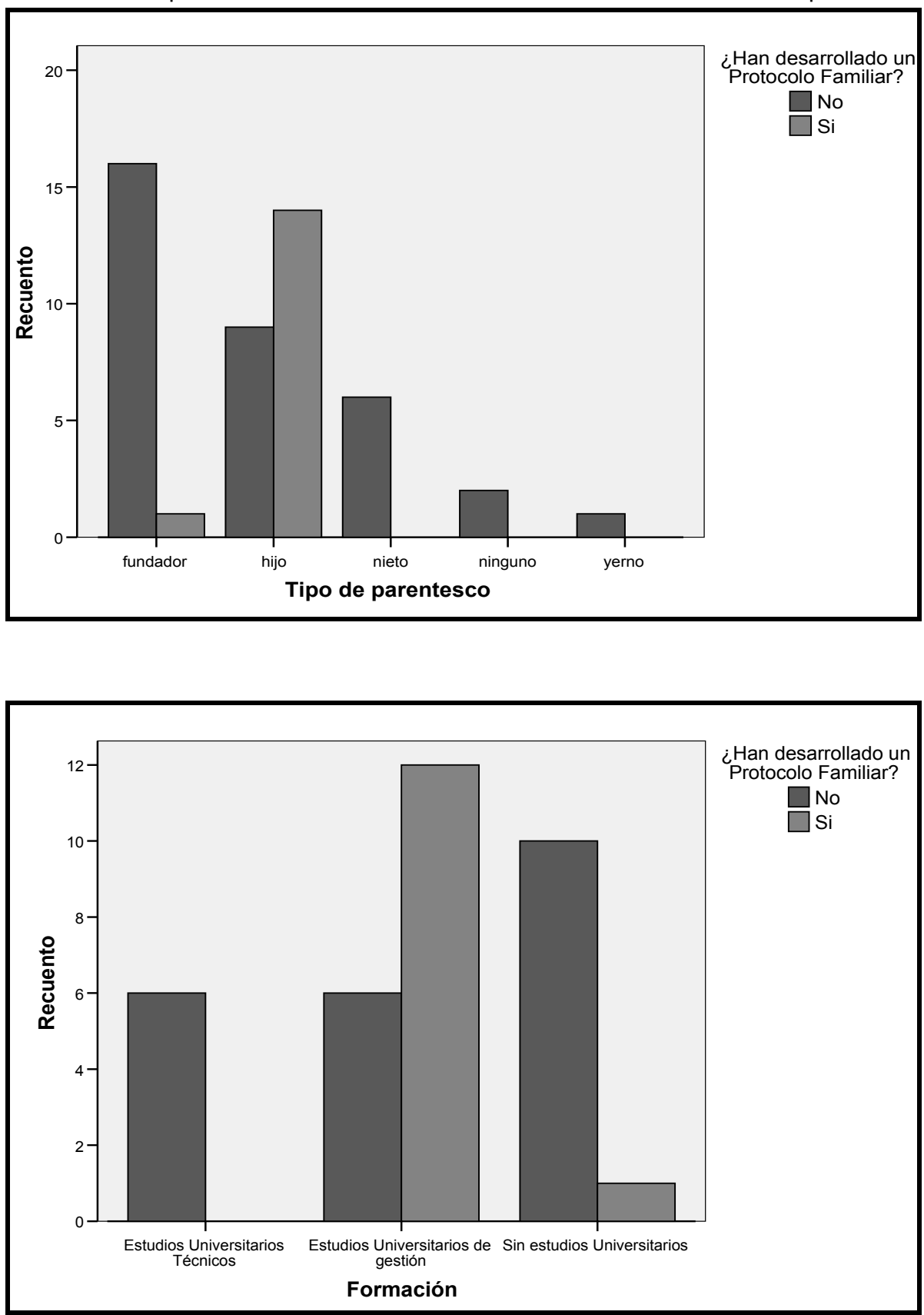


\section{TABLA 6 (continuación)}

Desarrollo del protocolo familiar frente a las características del máximo responsable.
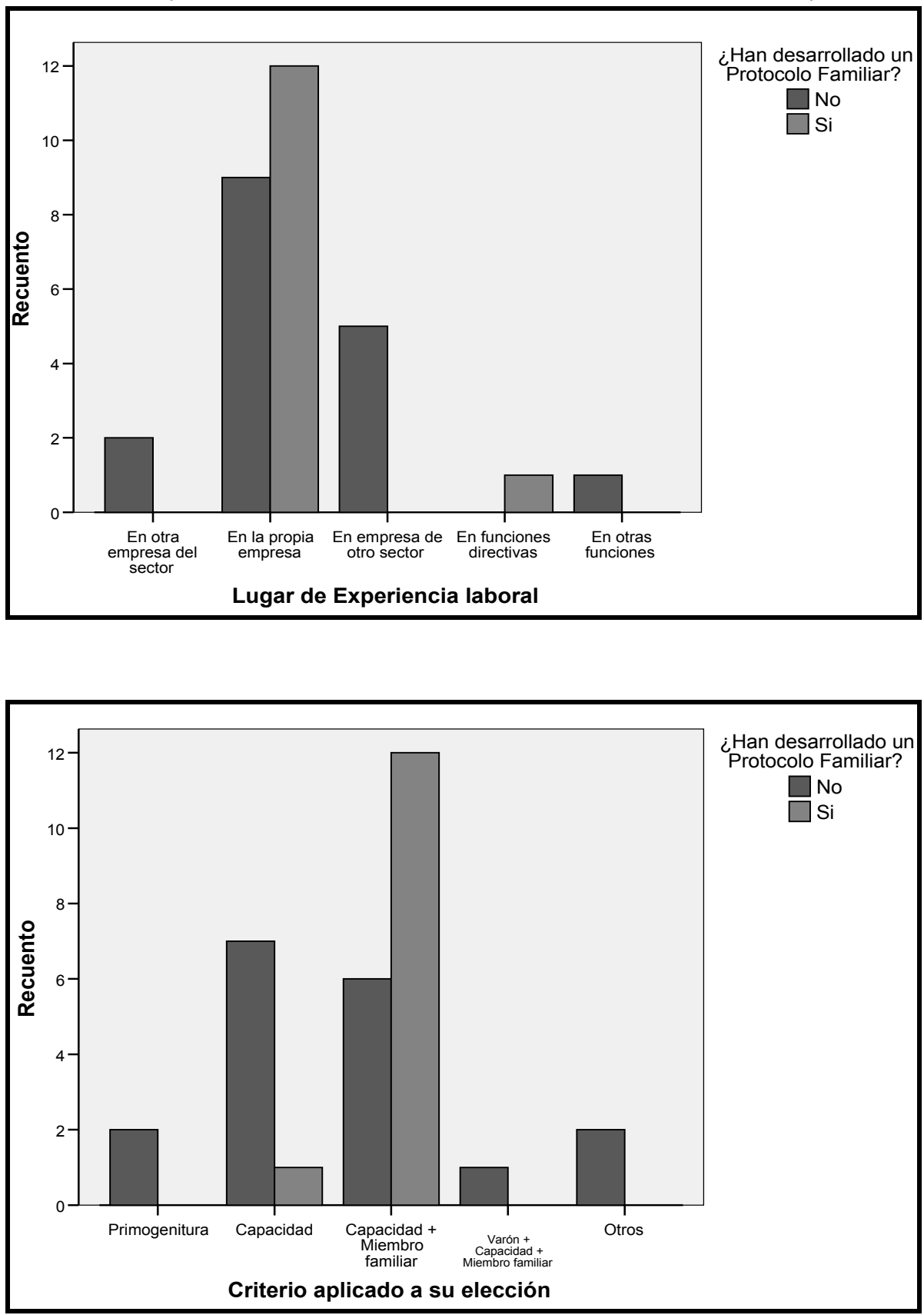
TABLA 6 (continuación)

Desarrollo del protocolo familiar frente a las características del máximo responsable.
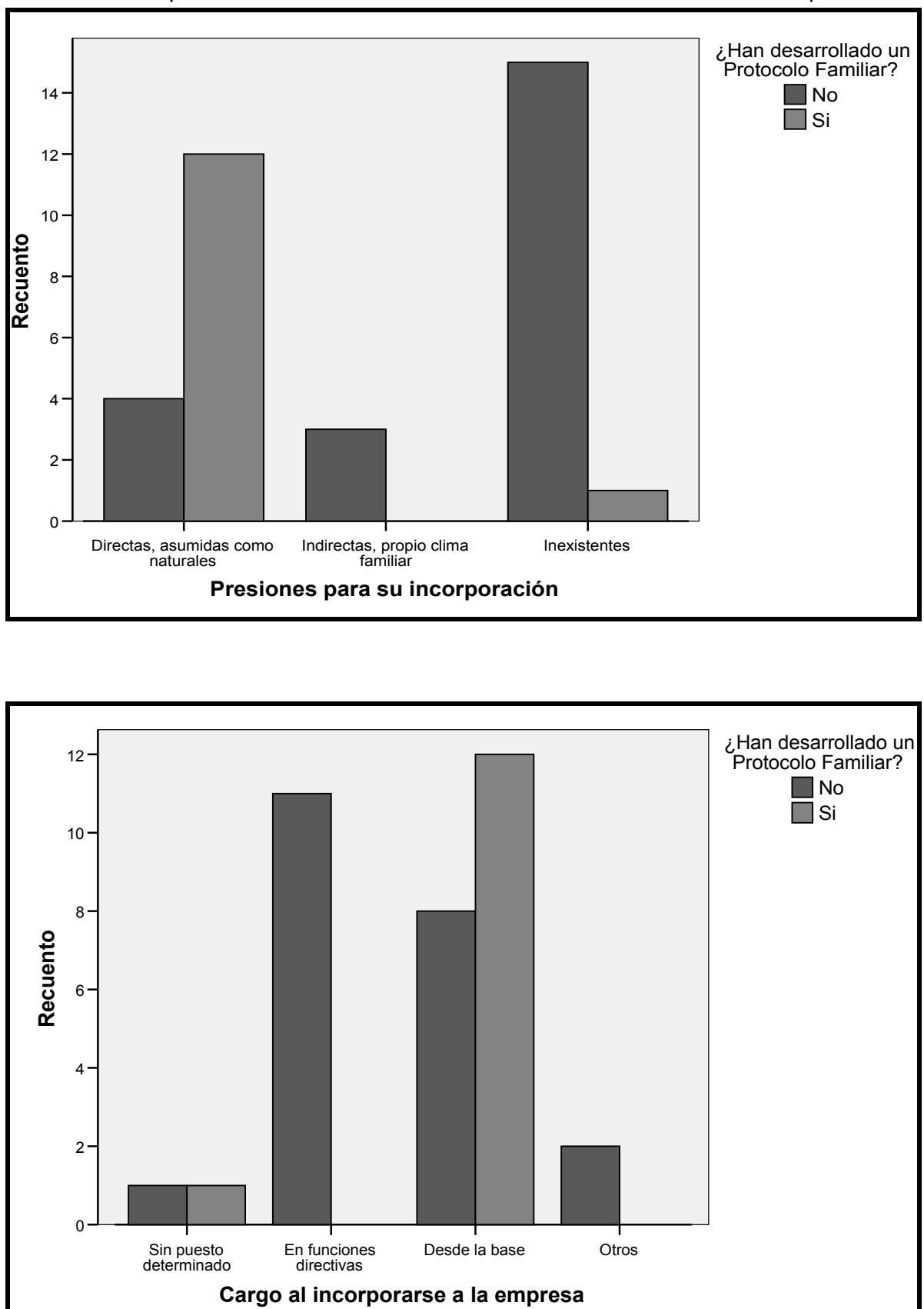

¿Han desarrollado un Protocolo Familiar? $\square$ No

$\mathrm{Si}$ 
TABLA 7

Influencia de las características del actual responsable de la empresa.

\begin{tabular}{|c|c|c|c|c|}
\hline Característica & V de Crámer & Significación & $\begin{array}{l}N \text { casos } \\
\text { válidos }\end{array}$ & $\begin{array}{l}\text { Hipótesis de } \\
\text { asociación }\end{array}$ \\
\hline $\begin{array}{l}\text { Presiones para su } \\
\text { incorporación }\end{array}$ & 0,720 & 0,000 & 35 & Válida \\
\hline Formación & 0,632 & 0,001 & 35 & Válida \\
\hline Tipo de parentesco & 0,619 & 0,001 & 49 & Válida \\
\hline $\begin{array}{l}\text { Criterio aplicado a su } \\
\text { elección }\end{array}$ & 0,595 & 0,027 & 31 & Válida \\
\hline $\begin{array}{l}\text { Cargo al incorporarse } \\
\text { en la empresa }\end{array}$ & 0,593 & 0,006 & 35 & Válida \\
\hline Experiencia laboral & 0,276 & 0,102 & 35 & No válida \\
\hline $\begin{array}{l}\text { Lugar de experiencia } \\
\text { laboral }\end{array}$ & 0,549 & 0,060 & 30 & No válida \\
\hline $\begin{array}{l}\text { Quién lo eligió como } \\
\text { sucesor }\end{array}$ & 0,490 & 0,059 & 31 & No válida \\
\hline
\end{tabular}

Dentro de estas características, no se pudo establecer una asociación entre la experiencia laboral, lugar de experiencia, ni persona que lo designó, con respecto a la existencia de un protocolo, ya que la significación de los resultados es insuficiente. Sin embargo, a la vista de las gráficas, y de que la hipótesis alternativa tampoco es concluyente, no se puede afirmar la independencia entre factores. Por ello, lo que se detecta es la incapacidad, con las respuestas dadas, de extraer una conclusión sobre la existencia o no de relación.

Sin embargo sí se ha encontrado una asociación entre otros factores consultados en la encuesta y la existencia del protocolo, que se resumen en la tabla 7 ordenados según el grado de asociación. Así se observa que la existencia de presiones para la incorporación a la empresa se asocia con la existencia del protocolo familiar; con el desarrollo de estudios universitarios de gestión, frente a estudios técnicos o falta de estudios; con empresas en las cuales ya se haya producido un proceso de sucesión en la empresa, y por tanto se encuentren en la segunda generación; con que a la hora de la designación del actual director haya importado el criterio de pertenencia al grupo familiar frente a sólo el criterio de capacidad; y, por último, a que el actual director se haya incorporado a la empresa desde la base en lugar de comenzar por puestos de la alta dirección. 
TABLA 8

Sentido de la asociación de las características del máximo responsable con el desarrollo de un protocolo familiar.

\begin{tabular}{|l|l|}
\hline Característica & Existencia de protocolos asociados con \\
\hline Presiones para su incorporación & Directas, asumidas como naturales \\
\hline Formación & Estudios universitarios de gestión \\
\hline Tipo de parentesco & Hijo \\
\hline Criterio aplicado a su elección & Capacidad + Miembro familiar \\
\hline Cargo al incorporarse en la empresa & Desde la base \\
\hline
\end{tabular}

iv) Normas que regulan las relaciones familia-empresa: En relación con la existencia de normas que regulan las relaciones familia-empresa en las empresas familiares gallegas, se podrían destacar distintos aspectos relevantes, por ejemplo: ¿existe este tipo de normas en su empresa familiar?; si existen, ¿en qué generación comienzan a implantarse?; ¿están recogidas en algún documento? y si, a pesar de no estar recogidas en ningún documento, ¿son conocidas y aceptadas?

En las tablas 1 y 2 , se puede observar que en el $66 \%$ de las empresas familiares gallegas no existen normas que regulen las relaciones familia-empresa. En el 34\% restante estas normas se implantan, en el $81,3 \%$ de los casos, cuando se incorpora a la dirección de la empresa familiar la segunda generación. Además, estas normas están recogidas en algún tipo de documento en el 76,5\% de las empresas familiares gallegas que responden a la encuesta.

Es también destacable que, en los casos en los que estas normas no están recogidas en ningún tipo de documento, los empresarios familiares gallegos afirman que éstas son conocidas y aceptadas en el $100 \%$ de las empresas que responden a esta encuesta.

Se lleva a cabo un análisis de asociación de la variable: ¿han desarrollado un protocolo familiar?, con otras variables relacionadas con este núcleo temático. La tabla 9 recoge los valores del coeficiente phi calculados para determinar el grado de asociación. Como es lógico, se encuentra en todos los casos una asociación entre la existencia de normas para regular la relación familia empresa y el grado de desarrollo de los protocolos familiares. Así ocurre con los distintos ámbitos de aplicación de las normas consultados, que abarcan aspectos como la incorporación de miembros de la familia a la empresa, la política de distribución de dividendos, medidas encaminadas a la valoración de la compra-venta de acciones, la existencia de un consejo de familia o, la incorporación de la mujer a puestos directivos en la empresa familiar.

El grado de asociación entre empresas en las que existen normas para regular la relación familia-empresa y que hayan desarrollado un protocolo familiar es muy elevado, con el parámetro phi de asociación igual a 0,818 con significación óptima. 
La mayor o menor asociación entre el ámbito al que se refieran las normas y la existencia de protocolos familiares se puede estudiar a partir de la tabla siguiente que resume, de forma ordenada los valores del coeficiente de asociación phi entre las respuestas correspondientes a la encuesta. La significación de los resultados obtenidos es en todos los casos suficiente para aceptar la validez de la hipótesis de asociación.

TABLA 9

Coeficientes de asociación phi para la relación entre el desarrollo de protocolos familiares y la existencia de normas que regulen la relación familia-empresa.

\begin{tabular}{|l|c|c|}
\hline \multicolumn{1}{|c|}{ Existencia de normas o medidas para } & Phi & Significación \\
\hline Adopción de criterios de valoración en compraventa & 0,814 & 0,000 \\
de acciones & 0,760 & 0,000 \\
Permanencia en puestos de alto nivel & 0,687 & 0,000 \\
Mantenimiento de un buen clima familiar & 0,682 & 0,000 \\
Incorporación de familiares a la empresa & 0,628 & 0,000 \\
Política de distribución de dividendos & 0,524 & 0,000 \\
Evitar la dispersión del capital & 0,434 & 0,005 \\
Participación del capital en el consejo de adminis- \\
tración
\end{tabular}

\section{CONCLUSIONES}

Como conclusiones del trabajo realizado, se ha obtenido, por un lado, el grado de desarrollo de los protocolos familiares en Galicia, y por otro, una serie de rasgos compartidos por las empresas que los han desarrollado.

- El grado de desarrollo de los protocolos familiares en Galicia se podría calificar como bajo, a fin de cuentas no deja de ser de un 30,61\%, pero concordante con la situación española reflejada en el estudio de Amat (1998) citado en la primera parte del artículo. Entre las empresas que aún no lo han desarrollado, un $53,85 \%$ tiene previsto desarrollarlo en algún momento del futuro, por lo que es de esperar que con el tiempo se extienda más su desarrollo. Sin embargo sigue habiendo otro $46,15 \%$ que no tiene intención de realizarlo, bien por falta de conocimiento o porque no lo percibe como un instrumento lo bastante útil para su empresa.

- Las empresas que han desarrollado un protocolo familiar se pueden caracterizar como empresas grandes con un alto grado de control familiar. Además, comúnmente se trata de empresas que ya han experimentado un proceso de sucesión (se encuentran en segunda generación) y en la que los actuales directores han estado muy ligados desde su comienzo a la empresa. Así se observa 
que en estos casos, en la designación del actual director, primó el criterio de pertenencia al grupo familiar, realizaron estudios universitarios de gestión, claramente orientados a asumir funciones directivas en el futuro, y se incorporaron a la empresa desde la base.

- Por último, cabe destacar que el desarrollo de protocolos familiares se asocia con la adopción de normas que aseguren el control de la empresa por parte del grupo familiar. Entre éstas, cabe destacar aquellas orientadas al control de la compraventa de acciones o la permanencia en puestos de alto nivel.

\section{REFERENCIAS BIBLIOGRÁFICAS}

AMAT, J. (1998): La continuidad de la empresa familiar, Ed. Gestión 2000, Barcelona.

AMAT, J. Y CORONA, J. F. (2007): El protocolo familiar. La experiencia de una década, Colección Instituto de Empresa Familiar, Deusto, Barcelona.

BARBEITO ROIBAL, S. (2002): El Proceso De Sucesión En La Empresa familiar Gallega: Análisis Empírico Y Conclusiones, tesis doctoral, A Coruña.

BARBEITO ROIBAL, S. et al. (2004): Análisis Descriptivo Y Propuestas Sobre El Proceso De Sucesión En La Empresa familiar Gallega, Tórculo Ediciones, A Coruña.

BARBEITO ROIBAL, S. et al. (2004): Revisión Bibliográfica. La Investigación Sobre Empresa familiar, Tórculo Ediciones, A Coruña.

BARBEITO ROIBAL, S. et al. (2004): "Visión europea del proceso de sucesión en la empresa familiar", Boletín Económico de ICE, n² 2821, pp. 27-37.

BARBEITO ROIBAL, S. et al. (2006): "Importancia de algunas características relevantes de los sucesores en las empresas familiares gallegas", Boletín Económico de ICE, $\mathrm{n}^{\circ} 2884$, pp. 43-54.

BARBEITO ROIBAL, S. et al. (2006): "O criterio de elección do sucesor nas empresas familiares galegas", Revista Galega de Economía, vol. 15, n² 2, pp. 1-16.

BARBEITO ROIBAL, S. et al. (2006): "Planning for the succession process among Galician family businesses", Economics Analysis Working Papers, vol. 5, $\mathrm{n}^{\circ} 10$, Disponible en: http://www.economistascoruna.org/eawp/

BARNES, L. B. (1998): "Incongruent hierarchies: Daughters and younger sons as company CEOs", Family Business Review, no 1 (1), pp. 9-21.

BARNES, L. B. \& HERSHON, S. A. (1976): "Transferring power in the family business", Family Business Review, $\mathrm{n}^{\circ}$ 54, pp. 105-114.

BERAZA GARMENDIA, A. (2000): "El protocolo como instrumento de garantía de la continuidad de la empresa familiar", Revista de dirección y administración de empresas, $\mathrm{n}^{\circ} 8$, Sept., pp. 17-27.

Boletín Oficial de las Cortes Generales-Senado. VII Legislatura. Serie I: Boletín general, n०312, de 23 de Noviembre de 2001, pp. 1-40.

BOE n 65 (2007): Real Decreto 171/2007, 16 Marzo de 2007, pp. 11254-11257.

CABRERA SUÁREZ, K. (1998): Factores determinantes del éxito y fracaso del proceso de sucesión en la empresa familiar, Tesis Doctoral, Las Palmas de Gran Canaria.

CHRISMAN, J.J. et al. (1998): "Important attributes of successor in family businesses: an exploratory study", Family Business Review, n 11, pp. 19-34.

DOCE, L385, de 31 de Diciembre de 1994, pp. 14 y ss.

DROZDOW, N. \& CARROL, V. P. (1997): "Tools for strategy Development in family firms", Sloan Management Review, Fall, pp. 75-88.

FERNÁNDEZ, Z. (1993): "La organización interna como ventaja competitiva para la empresa", Papeles de Economía Española, nº 56, pp. 178-193. 
FERNÁNDEZ-TRESGUERRES GARCÍA, A. (2002): "Protocolo Familiar: un instrumento para la autorregulación de la sociedad familiar", Revista de Derecho de Sociedades, $\mathrm{n}^{\circ} 19$.

FILLIS, I. (2001): "Small firm internationalisation: an investigative survey and future research directions", Management Decision, vol. 39,n 9, pp. 767-783(17).

GIBB DYER, W. JR. \& SÁNCHEZ, M. (1998): "Current State of Family Business Theory and Practice as Reflected", Family Business Review, n 11 (4), pp. 287-295.

GONZALEZ DE ZULUETA, F. y PAISÁNT, P. (2007): "El protocolo familiar: los retos de su publicidad", Economist \& Jurist, n 111, año XV, Junio 2007, pp. 40-45.

GORTÁZAR LORENTE, C. (2004): Les relacions económiques en la crisi familiar, Jornades de Dret Catalá a Tossa, Segona Artículo.

GRANT, P. \& PERREN, L. (2002): "Small Business and Entrepreneurial Research: Metatheories, Paradigms and Prejudices", International Small Business Journal.2002, $\mathrm{n}^{\circ} 20$, pp. 185-211.

HANDLER, W. C. (1992): “The succession experience of the nest generation”, Family Business Review, $\mathrm{n}^{\circ} 5$ (3), pp. 283-307.

LANSBERG, I. \& ASTRACHAN, J. H. (1994): "Influence of family relationships on succession planning and training: the importance of mediating factors", Family Business Review, $\mathrm{n}^{\circ} 7$ (1), pp. 39-59.

MARTÍNEZ DÍEZ, R (DIR) (2006): El patrimonio familiar, profesional y empresarial: su formación, protección y transmisión, Thomson-Civitas, Madrid.

NUENO, P (1999): "El lado enfermizo de la empresa familiar", Actualidad Económica, n 2135, p. 62.

OLSON, H. (2001): Executive summary: Should your next CEO be a woman? Center for Ethical Business Cultures, Disponible en: http://www.cebcglobal.org/Publications/Executive_summary/exs_0301.htm.

PONT CLEMENTE, J. F. (1998): "Mitos y paradojas del Protocolo Familiar", Alta Dirección, $n^{\circ} 33$, Nov-Dic, pp. 15-19.

RODRÍGUEZ ALCALDE, J. J. y RODRÍGUEZ ZAPATERO, M. (2007): El protocolo familiar: un juego de promesas y una cascada de contratos, colección Cátedra Prasa, Córdoba.

ROSENBLATT et al. (1985): The family in business: Understanding and dealing with the challengers entrepreneurial families face, CA: Jossey-Bass, San Francisco.

SANCHO, C. (2005): "Control y sucesión en la Empresa Familiar", Harvard Deusto Finanzas y Contabilidad, $\mathrm{n}^{\circ} 63$, Ene-Feb, pp. 26-34.

SCHIFF ESTESS, P. (2001): Women are taking over the family business: Their initiative is key; Entrepreneur, Disponible en: http://www.entrepreneur.com/mag/article/0,1539,286790,00.html.

SHARMA, P. et al. (1997): "Strategic management of the family business: Past research and future challenges", Family Business Review, n 10, pp. 1-35.

STRAVROU, E. (1999): "Succession in family businesses: exploring the effects of demographic factors on off-spring intention to join and take over the business", Journal of small business management, $\mathrm{n}^{\circ} 37$ (3), pp. 43-61.

VILANOVA, A. et al. (1999): Cómo elaborar un plan estratégico en la empresa. La empresa familiar, ESADE y Cinco Días, cap. 14.

WARD, J. L. (1993): Protocolo Familiar, La empresa familiar 4, IESE. 